\title{
Disruption of transitional stages in 24-h blood pressure recording in renal transplant recipients
}

\author{
Marcelo E. Katz ${ }^{1,2}$, Fernando Margulis ${ }^{3}$, Rubén Schiavelli ${ }^{3}$, Pablo Arias ${ }^{4}$, Geoffrey A. Head ${ }^{5}$ and \\ Diego A. Golombek ${ }^{1 *}$
}

' Department of Science and Technology, University of Quilmes, Buenos Aires, Argentina

${ }^{2}$ D. F. Santojanni Hospital, Buenos Aires, Argentina

${ }^{3}$ Dialysis and Kidney Transplantation Unit, Dr. Cosme Argerich Hospital, Buenos Aires, Argentina

${ }^{4}$ Department of Physiology, School of Medicine, University of Buenos Aires, Buenos Aires, Argentina

${ }^{5}$ Neuropharmacology Laboratory, Baker IDI Heart and Diabetes Institute, Melbourne, VIC, Australia

Edited by:

Ahmed BaHammam, King Saud

University, Saudi Arabia

\section{Reviewed by:}

F. Javier Puertas, University of

Valencia, Spain

Hani M. Lababidi, King Fahad Medical

City, Saudi Arabia

\section{*Correspondence:}

Diego A. Golombek, Departamento de Ciencia y Tecnología, Universidad

Nacional de Quilmes, Roque Sáenz

Peña 352, Bernal (B1876BXD),

Buenos Aires, Argentina.

e-mail:dgolombek@unq.edu.ar
Patients with kidney replacement exhibit disrupted circadian rhythms. Most studies measuring blood pressure use the dipper/non-dipper classification, which does not consider analysis of transitional stages between low and high blood pressure, confidence intervals nor shifts in the time of peak, while assuming subjective onsets of night and day phases. In order to better understand the nature of daily variation of blood pressure in these patients, we analyzed $24 \mathrm{~h}$ recordings from 41 renal transplant recipients using the non-symmetrical double-logistic fitting assessment which does not assume abruptness nor symmetry in ascending and descending stages of the blood pressure profile, and a cosine best-fitting regression method (Cosinor). Compared with matched controls, double-logistic fitting showed that the times for most transitional stages (ascending systolic and descending systolic, diastolic, and mean arterial pressure) had a wider distribution along the 24-h. The proportion of individuals without daily blood pressure rhythm in the transplanted group was larger only for systolic arterial pressure, and the amplitude showed no significant difference. Furthermore, the transplant recipient group had a less pronounced slope in descending systolic and ascending mean blood pressure. Cosinor analysis confirmed this phase-related changes, showing a wider distribution of times of peak (acrophases). We conclude that daily disruptions in renal transplant recipients can be explained not necessarily by an absence in diurnal variation, but also by changes in waveform-related parameters of the rhythm, and that alterations in the phase of the rhythm are the most consistent finding in these patients.

Keywords: daily rhythm, ambulatory blood pressure monitoring, kidney transplantation, non-symmetrical doublelogistic analysis, Cosinor

\section{INTRODUCTION}

It has long been known that patients subjected to organ replacement exhibit disruptions in blood pressure diurnal variations. This is mainly related to the absence of a nocturnal decrease in blood pressure, that has been found to be a predictor of hypertension and several chronic cardiopathies (Lipkin et al., 1993; van de Borne et al., 1993; Textor et al., 1994; Taler et al., 1995, 2000; van den Dorpel et al., 1996; Galiatsou et al., 2000; Curtis, 2002). Sleep disturbances are also very frequent in these patients, strongly affecting their quality of life (Neuhaus et al., 1994; Kemper et al., 2003). However, the mechanisms underlying these temporal disturbances have not been elucidated (van de Borne et al., 1993; Textor et al., 1994; Taler et al., 1995; van den Dorpel et al., 1996; Kooman et al., 2001; Curtis, 2002). Indeed, the control of the 24 -h variation of blood pressure is complex and, in some cases, controversial, since it is modulated by both endogenous and exogenous cues (Halberg et al., 1965; Degaute et al., 1991; Portaluppi et al., 1996; Witte et al., 1998), and influenced by other physiological and pathological variables like normal and pathological sleep (Smolensky et al., 2007), including obstructive sleep apnea syndrome (Lusardi et al., 1996; Kario, 2009) and neuroendocrine parameters such as cortisol, sympathetic mediators, and the renin-angiotensin-aldosterone system, all of which are indeed modulated by the circadian clock (Holt-Lunstad and Steffen, 2007; Rudic and Fulton, 2009). In addition, humoral factors that affect blood pressure directly, such as renin, are also dependent on sleep stages (Brandenberger et al., 2005).

Although an accurate description of these temporal disruptions is necessary in order to determine worthwhile associations, not all of the data have been analyzed with specific chronobiological tools. Sleep disturbances have been studied with ad-hoc questionnaires and the daily rhythm of blood pressure and heart rate have been analyzed following the standard classification of dippers/non-dippers (D/ND). This classification, defined as a difference between diurnal and nocturnal average levels of more or less than $10 \%$, respectively, does not consider transitional periods between low and high blood pressure stages, confidence intervals, changes in the waveform or shifts in the phase of blood 
pressure rhythm. Moreover, it does not analyze diurnal and nocturnal values with precision as a result of a subjective definition of day and night onsets (Parati, 2004).

The non-symmetrical double-logistic fitting procedure for modeling 24-h blood pressure variation (Head et al., 2004) is based on the assumption that transitions between lower and higher levels of blood pressure are not abrupt and symmetrical, and are independent from subjective definitions of awake and asleep stages. This method has been clearly validated in a number of physiological and pathological situations (Head et al., 2005, 2006, 2010). In order to perform a more detailed analysis of the daily disruptions in blood pressure in renal transplant recipients, we have performed a retrospective study to apply this double-logistic fitting to 24-h blood pressure recordings of a group of patients subjected to kidney replacement, as compared to a group of subjects matched by age, sex, and the average of 24 -h mean arterial pressure (MAP). In addition, we have also applied the Cosinor method, which determines the best-fitting cosine waveform to the data in terms of period, amplitude, and time of peak (acrophase) (Nelson et al., 1979; Bingham et al., 1982; Mohri et al., 2003). We also analyzed the associations of daily disruptions with pharmacological and physiological parameters.

\section{MATERIALS AND METHODS PATIENTS}

In this retrospective study, the ambulatory blood pressure monitoring $(\mathrm{ABPM})$ data of adult renal transplant recipients was analyzed in order to assess their $24 \mathrm{~h}$ profile. Patients diagnosed with any cardiovascular disease other than hypertension, with neurological or psychiatric diseases, night workers, and those with sleep complaints, were excluded from the study. Twenty-four-hour records with more than one-third of data absent were not used for analysis. Forty-one renal transplant recipients were included, all of them were receiving oral treatment with CsA and meprednisone, 34 patients were also receiving mycophenolate mofetil, while the remaining 7 were on azathioprine treatment; 27 were hypertensive according to the Joint National Committee on Prevention, Detection, Evaluation, and Treatment of High Blood Pressure (JNC 7; Jones and Hall, 2004) and were receiving antihypertensive drugs. For the control group, 41 non-transplanted individuals that were receiving medical attention at the Internal Medicine department of the same hospital were enrolled in the study, matched by sex, age, and the average of $24 \mathrm{~h} \mathrm{MBP}$ (24 MAP-av), with the same exclusion criteria. All the individuals' filiation data included in the study were blind for the researchers that performed the analysis. Informed consent was obtained from all subjects. The ethics committees of the University of Quilmes, Buenos Aires, Argentina and the Dr. Cosme Argerich Hospital, Buenos Aires, Argentina, approved the study.

\section{AMBULATORY BLOOD PRESSURE MONITORING}

Ambulatory blood pressure monitoring was recorded noninvasively over $24 \mathrm{~h}$ on a regular working day with an Accutraker II (Suntech Medical Inc., NC, USA) with the cuff placed in the arm without arteriovenous fistula. Recordings were made every $15 \mathrm{~min}$ during daytime (08:00 a.m. to 11:00 p.m.) and every 20 min during the night (11:00 p.m. to 08:00 a.m.). Individualized analysis of ambulatory blood pressure profiles was performed by adjusting readings according to the awake/asleep written report of each participant, who were instructed to sleep at their usual time (sleep onset 09.00 to 11.00 p.m.). The average $24 \mathrm{~h}$, awake and asleep blood pressures were also recorded.

\section{MEASUREMENTS OF SERUM CREATININE, CREATININE CLEARANCE, AND SERUM CYCLOSPORIN}

Data was collected on the day of the blood pressure monitoring during the morning, before breakfast, and before the morning dose of CsA $\left(T_{0}\right)$. The Jaffé method was used for measurement of both serum and urinary creatinine. Creatinine clearance was calculated by the formula $U / S \times$ urine flow ( $U=$ urinary creatinine concentration, $S=$ serum creatinine concentration). Serum CsA was measured by a fluorescence polarization immunoassay (Abbott Laboratories, IL, USA).

\section{MODELING OF 24-h PROFILE OF BLOOD PRESSURE}

The double-logistic method was applied as described (Head et al., 2004). This model considers a stage of high level of blood pressure, named high plateau, a stage of low level of blood pressure, named low plateau, the range of the variation, quantified as the difference between high and low plateaus, and two transitional stages modeled logistically. These transitional stages are calculated independently, relating to separate ascending and descending parameters. The time of each transition corresponds to the time when the curve reaches the $50 \%$ of its final value (Figure 1). This method also calculates the slope of the variation. Fittings with a $p$ value $>0.05$ were considered as non-statistically significant and therefore without daily variation. The parameters defined by the model were analyzed only in individuals with significant daily variation. In Table 2 , time of transition stages are shown in numerical format (100 decimals $=60 \mathrm{~min})$.

The Cosinor analysis was performed as described previously (Nelson et al., 1979; Bingham et al., 1982; Mohri et al., 2003). This method consists of adjusting the best least-squares fitting cosine waveform to the data according to the equation $y=M+A \cos$ $(f+\omega t)$, in which $M$ corresponds to mesor (mean estimated over rhythm), $A$ is the amplitude which is defined as the value that corresponds to half of the difference between the maximum-minimum values of the adjusted curve, $t$ is time, $\omega$ is angular velocity that, in a 24 -h-period rhythm, corresponds to $2 \pi / 24=0,2618$ radians $/ \mathrm{h}$ (with time expressed in hours), and $f$ represents the acrophase (time of peak) which is converted into hours dividing it by $\omega$. Waveform fittings with a $p$ value $>0.05$ were considered as non-statistically significant and therefore without daily variation. Amplitude and acrophase were analyzed only in individuals with significant daily variation (Figure 1). In Table 3, acrophase is showed in numerical format (100 decimals $=60 \mathrm{~min})$.

\section{STATISTICAL ANALYSIS}

Data were averaged into $1 \mathrm{~h}$ bins for smoothing of serial recordings (sampling frequency was different between day and night). All comparisons of proportions (like analysis of fitting-related variables in patients with or without hypertension) were made with Fisher's exact test. Differences between groups were analyzed with either Student's, Welsh's, or Mann-Whitney's test according to the 

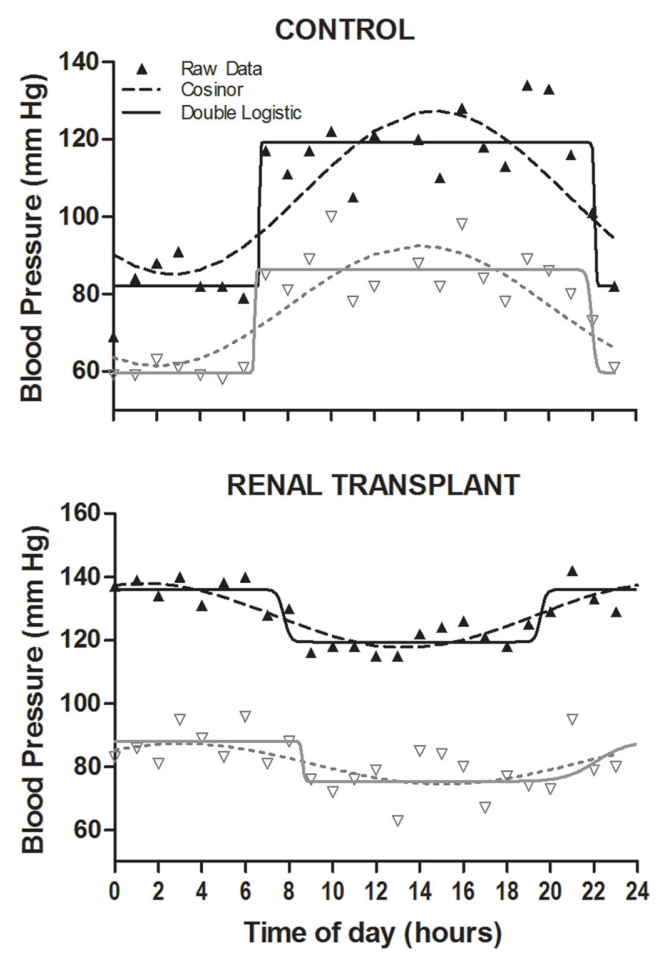

FIGURE 1 | Individual examples of the modeling procedures in both groups. The upper plot corresponds to a control individual and the lower plot to a renal transplant recipient. Double-logistic fitting is plotted with solid line and Cosinor fitting with dash-dot line. Black filled triangles are SAP hourly values and white filled triangles DAP values.

assessment of normality distribution of data which was measured with the combination of Shapiro-Wilk test and the visual examination of Normality and Box plots by at least two observers, and the comparison of SDs with F test when appropriate. Differences in the distribution of data (variances) were analyzed with the Modified Levene's test (Neter et al., 1996). Since most of numerical comparison were performed with non-parametric tests, the data is presented as median (first quartile; third quartile) in all cases, for homogenization. Differences were considered as statistically significant when $p<0.05$.

\section{RESULTS}

The most relevant characteristics of both groups are summarized in Table 1.

\section{PRESENCE OF A DAILY VARIATION ACCORDING TO THE DOUBLE-LOGISTIC METHOD}

To assess the presence of a daily rhythm in 24-h blood pressure variations, we compared the proportion of individuals whose double-logistic fitting of 24-h blood pressure profile reached statistical significance. Differences between patients and controls were significant for systolic arterial pressure (SAP), with a lower proportion of renal transplant recipients showing statistically significant rhythms, but did not reach significance for diastolic arterial pressure (DAP) or MAP (Table 2).
Table 1 | Characteristics of renal transplant recipients and controls.

\begin{tabular}{|c|c|c|}
\hline & Renal transplant recipients & Controls \\
\hline Number of subjects & 41 & 41 \\
\hline Sex, female/male & $17 / 24$ & $17 / 24$ \\
\hline Age, years & $43(18 ; 32 ; 50 ; 69)$ & $42(20 ; 32 ; 50 ; 69)$ \\
\hline $\begin{array}{l}\text { Average of } 24 \mathrm{~h} \mathrm{MAP} \text {, } \\
\mathrm{mmHg}\end{array}$ & 101 (78.7; 93.8; 109.3; 132) & $\begin{array}{l}100.7 \text { (79; 93.3; } \\
108.1 ; 134.7)\end{array}$ \\
\hline TESS, months & $26(2 ; 7.5 ; 59 ; 192)$ & \\
\hline \multicolumn{3}{|l|}{ Cyclosporin } \\
\hline Oral dose, mg/kg & $2.77(0.81 ; 1.86 ; 3,54 ; 5.32)$ & \\
\hline $\begin{array}{l}\text { Serum concentration, } \\
\mathrm{ng} / \mathrm{dl}\end{array}$ & $144(25 ; 103 ; 176 ; 259)$ & \\
\hline Meprednisone, mg/kg & $0.09(0.04 ; 0.06 ; 0,12 ; 0.34)$ & \\
\hline $\begin{array}{l}\text { Mycophenolate mofetil, } \\
\mathrm{mg} / \mathrm{kg}(n=34)\end{array}$ & $28.1(13.3 ; 24.7 ; 32.9 ; 42.5)$ & \\
\hline $\begin{array}{l}\text { Azathioprine, } \mathrm{mg} / \mathrm{kg} \\
(n=7)\end{array}$ & $1.1(0.72 ; 0.82 ; 1.26 ; 1.35)$ & \\
\hline
\end{tabular}

Data is presented as median (minimum; first quartile; third quartile; maximum) except for number of subjects and sex. MBP, mean blood pressure; TESS, time elapsed since date of surgery.

\section{DIFFERENCES IN PARAMETERS ANALYZED BY THE DOUBLE-LOGISTIC METHOD}

In order to analyze the characteristics of 24 -h variations we compared statistically significant double-logistic fittings between renal transplant recipients and controls regarding the six parameters defined from the model. Renal transplant recipients exhibited increased levels of low plateaus for SAP, DAP and MAP, and of high plateau only in SAP. However, the amplitude of the daily variation remained unchanged for the three parameters (Table 2).

The analysis of the steepness of transition between high and low blood pressure plateaus showed that the rate (slope) of increase in MAP was slower for renal transplant recipients. Transition from high to low plateau remained unchanged.

In the case of the times when transitional periods occur, we found a significantly wider distribution along the 24-h for SAP and MAP ascending stage (Table 2; Figure 2). The same wider distribution was found for the time when SAP, DAP, and MAP descending stages occurred (Table 2; Figure 3). Indeed, in the representative examples of Figure 1 we chose to depict one of the patients that exhibited an inversion in the timing of blood pressure rhythms.

The non-parametrical comparison showed also that the time of DAP ascending occurred later in renal transplant patients

Since the time of transition is related to the phase of blood pressure rhythms, we performed the Cosinor analysis to confirm this phase-related disorder.

\section{COSINOR ANALYSIS}

Although in all cases the proportion of individuals with a significant daily variation according to Cosinor analysis was higher for controls, the differences found in SBP, DBP, and MBP did not reach statistical significance (Table 3). However, renal transplant recipients exhibited a significantly lower amplitude of their SBP 
Table 2 | Comparison of parameters analyzed by double-logistic method between renal transplant recipients and controls.

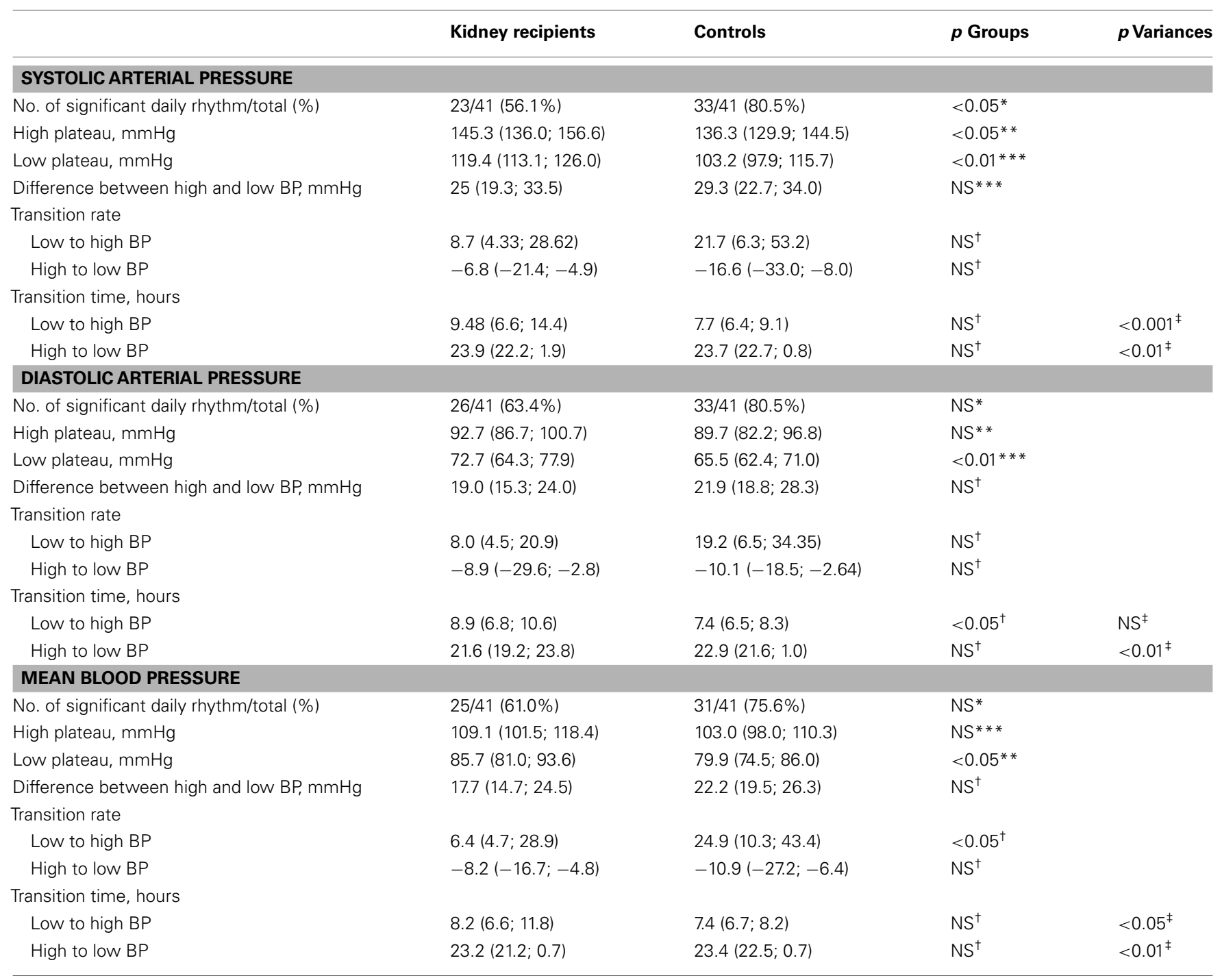

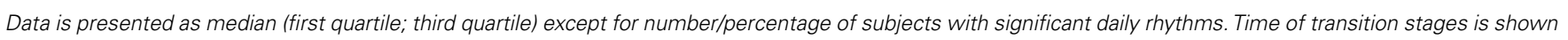

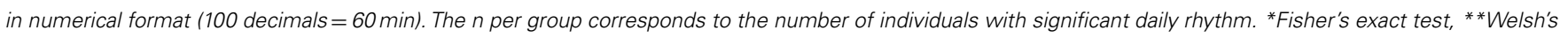
test, ${ }^{* *}$ *Student's test, ${ }^{+}$Mann-Whitney test, ${ }^{\ddagger}$ Modified Levene's test. BP, blood pressure; NS, not statistically significant.

and MBP rhythms (Table 3). As suggested by double-logistic fitting, the analysis of the acrophase, which reflects the phase of a daily variation, showed that its distribution along the 24-h is clearly narrower in the control group than in renal transplant recipients, who exhibited a significantly higher variance in the peak times of SBP, DBP, and MBP (Table 3).

\section{ASSOCIATION ANALYSIS OF THE DIURNAL PROFILE OF BLOOD PRESSURE IN RENAL TRANSPLANT RECIPIENTS}

In order to explain the disruptions found in double-logistic fittingrelated parameters in renal transplant recipients, we analyzed the association between them and several variables. We divided the kidney recipients group according to the level of oral dose of CsA, serum concentration of CsA, oral dose of meprednisone, treatment with either mycophenolate mofetil or azathioprine, the time elapsed since the date of the transplant to the date of the study, serum concentration and clearance of creatinine, age, and the average of the 24-MBP-av. An additional subdivision for the analysis relied on whether patients had been diagnosed with hypertension or not. In numerical variables we used the median of each variable as the cut-of value to generate high and low level sub-groups. We could not find any significant association. Multivariate analysis could not be performed because of the absence of variables that reached an association with a $p$ value less than 0.10 .

\section{DISCUSSION}

Given the extensive description of disruptions in day/night blood pressure variation in renal transplant recipients that have been made with the classical D/ND classification, we aimed to analyze daily blood pressure rhythm with two mathematical related modeling methods, in order to achieve a better understanding of the 
Table 3 | Comparison of parameters analyzed by Cosinor method between renal transplant recipients and controls.

\begin{tabular}{|c|c|c|c|c|}
\hline & Kidney recipients & Controls & $p$ Groups & $p$ Variances \\
\hline \multicolumn{5}{|l|}{ SYSTOLIC ARTERIAL PRESSURE } \\
\hline No. of significant daily rhythm/total (\%) & $27 / 41(65.9 \%)$ & $29 / 41(70.7 \%)$ & NS* & \\
\hline Amplitude, $\mathrm{mmHg}$ & $10.1(8.3 ; 12.9)$ & $15.8(11.0 ; 17.4)$ & $<0.05^{* *}$ & \\
\hline Acrophase, hours & $15.6(13.9 ; 20.2)$ & $15.5(14.4 ; 16.7)$ & $N S^{* *}$ & $<0.01 * * *$ \\
\hline \multicolumn{5}{|l|}{ DIASTOLIC ARTERIAL PRESSURE } \\
\hline No. of significant daily rhythm/total (\%) & $26 / 41(63.4 \%)$ & $33 / 41(80.5 \%)$ & $N S^{*}$ & \\
\hline Amplitude, mmHg & $8.4(6.4 ; 11.4)$ & $9.9(7.8 ; 12.3)$ & $N S^{* *}$ & \\
\hline Acrophase, hours & $14.4(13.3 ; 17.5)$ & $14.9(14.2 ; 16.1)$ & $N S^{* *}$ & $<0.05^{* * *}$ \\
\hline \multicolumn{5}{|l|}{ MEAN BLOOD PRESSURE } \\
\hline No. of significant daily rhythm/total (\%) & $27 / 41(65.9 \%)$ & $34 / 41(82.9 \%)$ & NS* & \\
\hline Amplitude, $\mathrm{mmHg}$ & $8.2(6.1 ; 9.8)$ & $10.4(7.7 ; 12.8)$ & $<0.05^{* *}$ & \\
\hline Acrophase, hours & $14.8(13.4 ; 17.8)$ & $15.1(14.2 ; 16.3)$ & $N S^{* *}$ & $<0.05^{* * *}$ \\
\hline
\end{tabular}

Data is presented as median (first quartile; third quartile) except for the percentage of subjects with significant daily rhythms. Acrophase is shown in numerical format $\left(100\right.$ decimals $=60 \mathrm{~min}$ ). The $n$ per group corresponds to the number of individuals with significant daily rhythm. ${ }^{*}$ Fisher's exact test, ${ }^{* *}$ Mann-Whitney's test, ***modified Levene's test. NS, not statistically significant.

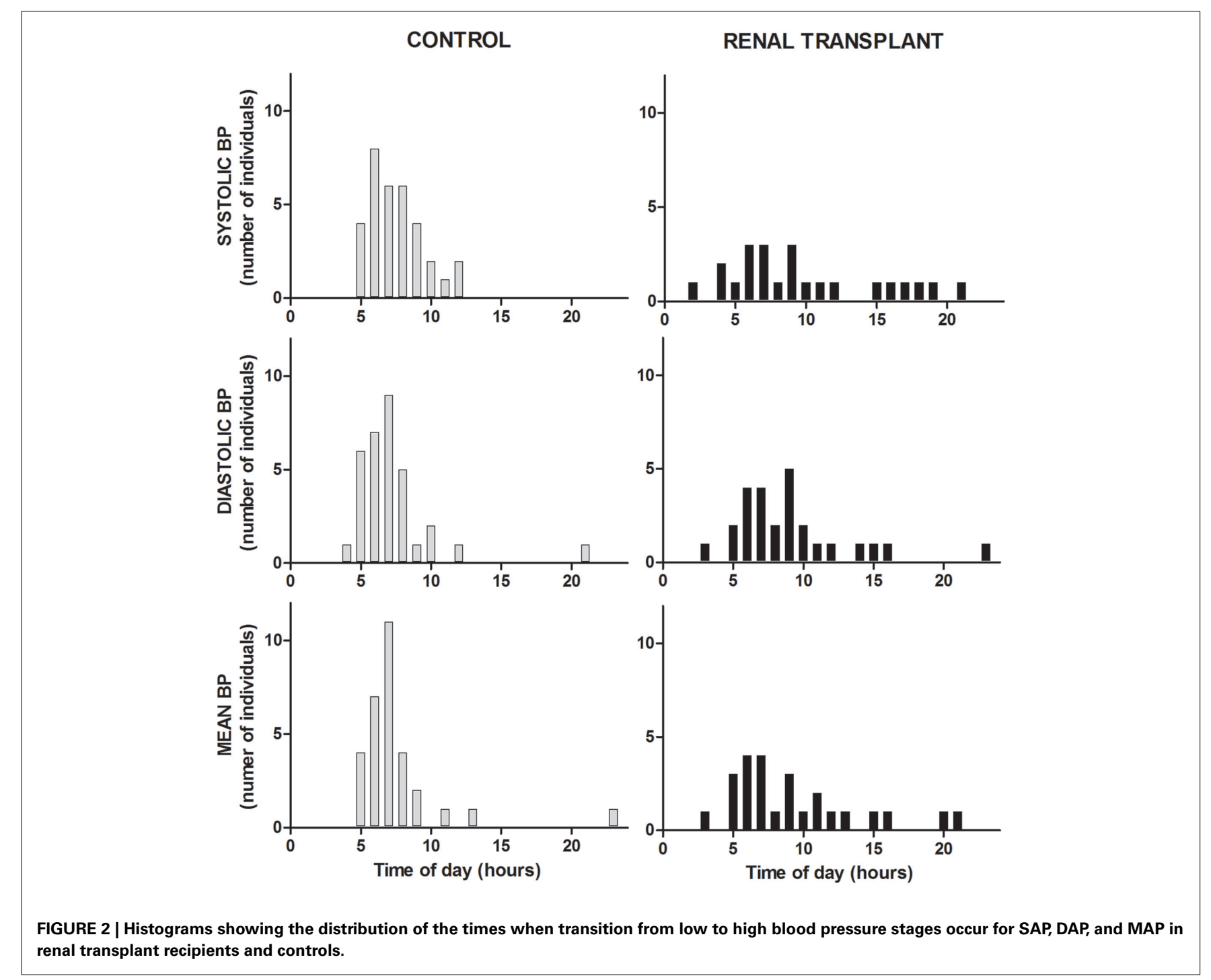


CONTROL
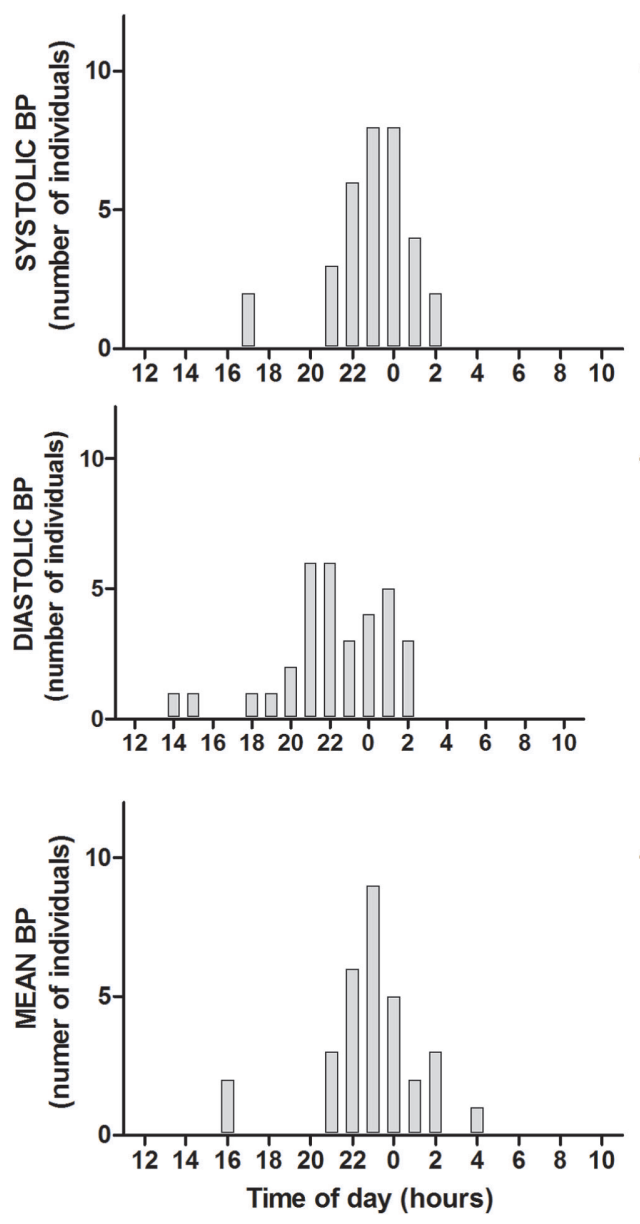

RENAL TRANSPLANT
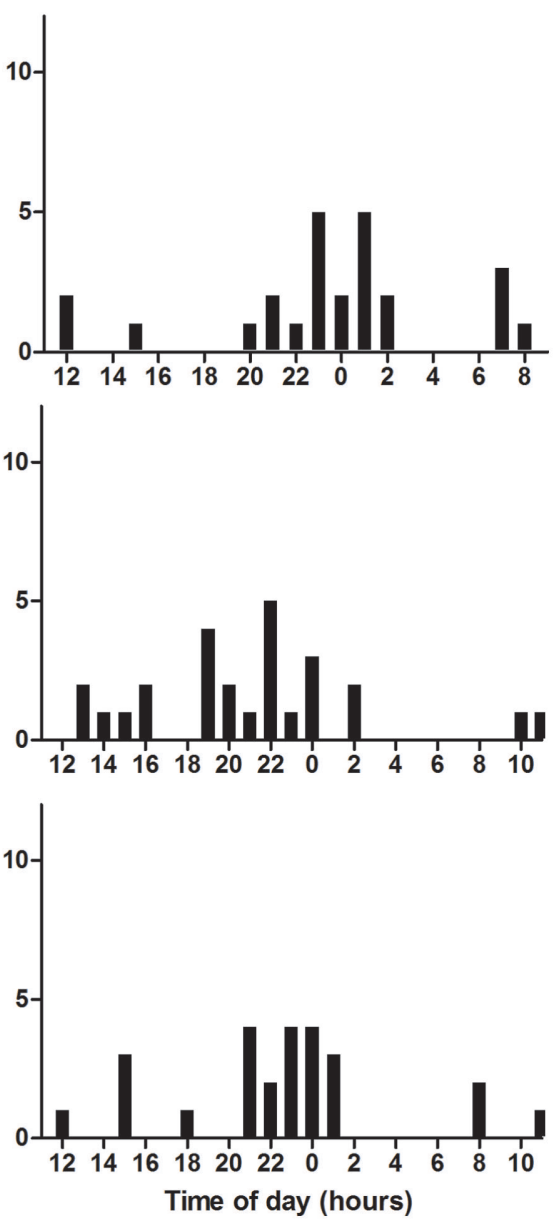

FIGURE 3 | Histograms showing the distribution of the times when transition from high to low blood pressure stages occur for SAP, DAP, and MAP in renal transplant recipients and controls.

nature of daily alterations in these patients: the double-logistic and the Cosinor methods. The first one, particularly, allowed the independent analysis of ascending and descending transitional periods. It was interesting to find that, despite what has been traditionally described with the D/ND classification, renal transplanted patients have blunted daily rhythms only for SAP, but did have consistent disruptions in waveform-related parameters such as time of transitional stages, and time of peak of the variation (depending on the method employed).

The wider distribution along the 24-h of the times of SAP and MAP ascending, and SAP, DAP, and MAP descending transitional stages in renal transplant recipients, as well as the slower ascending slope in MAP, clearly suggest a disruption in the mechanisms that rule the rise and decrease of blood pressure in kidney recipients. Some of the patients (as the one depicted in Figure 1) exhibited a clear inversion of temporal parameters of blood pressure. There are evidences of the compromise of the sympathetic nervous system in the normal regulation of the morning rise in blood pressure (Panza et al., 1991), and little is known about the mechanisms involved in the evening decline, even whether it is an active or a passive process. In renal transplant recipients, CsA has been particularly involved with sympathetic disturbances that induce a rise in blood pressure levels (Textor et al., 1994; Curtis, 2002), suggesting an active role of pharmacological treatment in disruptions in transitional stages. We could not find, however, any association of the waveform-related parameters with immunosuppressive treatment or any other pharmacological or physiological characteristics of patients. In particular, we found no association regarding hypertensive status; however, since hypertension is an important factor influencing blood pressure rhythm, additional studies should consider its role in more detail. Moreover, since neurohormonal factors (such as cortisol and renin secretion) influence blood pressure by several different mechanisms - some of which are sleep-dependent, its role in circadian disruption in renal transplant recipients should be acknowledged in more detail. It should be stated that the number of patients evaluated in this study could represent a limitation for the analysis, and might have masked an effect of medication or hypertensive status on blood pressure variation. Larger studies concerning the relevance of these cues need to be performed 
The presence of higher plateaus in renal transplant recipients is in accordance with previous descriptions of an increased rate of hypertensive status in renal transplant recipients (Textor et al., 1994; Curtis, 2002). Hypertension has also been associated to alterations in blood pressure rhythms, in particular with phase-related changes (Iantorno et al., 2003; Perez-Lloret et al., 2004). It is important to note that although both groups were matched by the average of the 24-h MAP, the comparisons of rhythmic parameters have been performed among patients with statistically significant daily rhythm, regardless of the initial matching protocol, so these differences are indeed representative of the changes present in the transplant recipient group.

In the case of the amplitude, we expected it to be reduced in kidney recipients, since the classical description of lower differences between day and night periods with the D/ND classification; however, no differences could be found between groups in our work. These results are in accordance with our finding that phase-related changes are the most consistent disruptions in kidney recipients.

Although the control group analyzed in this work differs in many aspects from the renal transplant group (which represents an organ replacement situation after a long time with an end-stage illness), albeit being matched for sex, age, and the average of $24 \mathrm{~h}$ MBP, these phase-related disruptions in the daily rhythm of blood pressure in transplanted individuals, taken together with the evidence of sleep disturbance (Neuhaus et al., 1994; Kemper et al., 2003), support the hypothesis of a weaker activity of the biological clock; moreover, the nature of these time-related disarrangements (wide distribution of time of ascending and descending blood pressure) can be explained as a blunted daily synchronization of endogenous rhythms to environmental cues. Supporting this hypothesis is the fact that the daily rhythm of blood pressure has a clear endogenous influence as described in its early morning rise (i.e., before waking time), its persistence in constant conditions, and its abolition after lesions of the hypothalamic suprachiasmatic nuclei (the central mammalian biological clock) (Halberg et al., 1965; Degaute et al., 1991; Portaluppi et al., 1996; Witte et al., 1998).

It is tempting to speculate that at least some of the changes exhibited in the renal transplant patients are related to their immune suppressant treatment. In our laboratory, we found not only the presence of calcineurin (the target of CsA and FK506) in the suprachiasmatic nuclei, but also that rodents treated with these immune suppressants have modifications in the activity of the circadian clock, as measured by locomotor activity rhythms, and a weaker synchronization to light stimuli (which resembles daily synchronization of the biological clock to day/night environmental cycle) (Katz et al., 2008). Indeed, it has been described that calcineurin inhibitors are capable of modifying electrical activity in the suprachiasmatic nuclei (Fukunaga et al., 2002). However, the lack of a clear association between circadian disruption

\section{REFERENCES}

Bingham, C., Arbogast, B., Guillaume, G. C., Lee, J. K., and Halberg, F. (1982). Inferential statistical methods for estimating and comparing cosinor parameters. Chronobiologia 9, 397-439.

and pharmacological treatment suggests that other mechanisms might be responsible for blood pressure rhythms alterations in renal transplant recipients. Notwithstanding, additional research is needed to determine the effect of clinical drugs on blood pressure variations, mainly because of the large list of factors that can influence this variable (Degaute et al., 1991; Lusardi et al., 1996; Portaluppi et al., 1996; Brandenberger et al., 2005).

As we have shown in this study, the double-logistic method allows a detailed analysis of the kinetics of BP rhythms, which could be useful to hypothesize about specific mechanisms related to the rise and decline in this parameter. There may be many cases were the $\mathrm{D} / \mathrm{ND}$ classification is a more effective tool in BP analysis; indeed, other mathematical regression models could also be relevant for this analysis (Chau et al., 1989; Degaute et al., 1991; Idema et al., 1992; Stanton et al., 1992). A comparative analysis in larger populations among different methods is mandatory in order to determine which is the best descriptor of the variation, in terms of biological and clinical relevant parameters like prediction of morbidity. Another limitation of our study is the lack of repeated measures ( $>24 \mathrm{~h}$ recording) in each individual, which could avoid hazardous collection of disarranged data due to intra-individual day by day variation. However the inter-individual reproducibility of shifted acrophase supports this finding. An additional limitation of the study is the lack of information concerning sleep performance in the population analyzed, since sleep disruption exerts an important influence on blood pressure (Chervin and Guilleminault, 1994; Lusardi et al., 1996). We can, however, discard sleep apnea on the basis of an initial questionnaire to patients and family regarding specific signs during the night.

In conclusion, the non-symmetrical double-logistic fitting and the Cosinor methods allowed us to get a more detailed description of disruptions occurring in blood pressure daily variations in renal transplant recipients. These analyses indicate that a phase-related disturbance occurs in these patients, rather than a complete loss of circadian variation. The traditional classification between dippers and non-dippers clearly differs from other chronobiological types of analysis when defining significant daily rhythmicity. The results found in this analysis have important clinical implications, which should be taken into account for predictive and therapeutic actions in kidney transplantation.

\section{ACKNOWLEDGMENTS}

The technical assistance of Machinandiarena, M. A., and Prato, C., in the determination of creatinine and cyclosporine levels is gratefully acknowledged. Supported by a fellowship from the University of Buenos Aires to Marcelo E. Katz. Diego A. Golombek is a member of the National Research Council (CONICET). The contribution from GAH was supported by the NHMRC and in part by the Victorian Government's Operational Infrastructure Support Program.

O., and Mathern, G. (1989). Twentyfour-hour ambulatory blood pressure in shift workers. Circulation 80, 341-347.

Chervin, R. D., and Guilleminault, C. (1994). Ambulatory monitoring of blood pressure in patients with sleep-disordered breathing. J. Cardiovasc. Risk 1, 127-131.

Curtis, J. J. (2002). Hypertensinogenic mechanism of the calcineurin inhibitors. Curr. Hypertens. Rep. 4, 377-380. 
Degaute, J. P., Van De Borne, P., Linkowski, P., and Van Cauter, E. (1991). Quantitative analysis of the 24-hour blood pressure and heart rate patterns in young men. Hypertension 18, 199-210.

Fukunaga, K., Horikawa, K., Shibata, S., Takeuchi, Y., and Miyamoto, E. (2002). Ca2+/calmodulin-dependent protein kinase II-dependent long-term potentiation in the rat suprachiasmatic nucleus and its inhibition by melatonin. J. Neurosci. Res. 70, 799-807.

Galiatsou, E., Morris, S. T., Jardine, A. G., Rodger, R. S., Watson, M. A., and Elliott, H. L. (2000). Cardiac and vascular abnormalities in renal transplant patients: differential effects of cyclosporine and azathioprine. J. Nephrol. 13, 185-192.

Halberg, F., Siffre, M., Engeli, M., Hillman, D., and Reinberg, A. (1965). Free-run study of circadian rhythms of the pulse, of waking-sleep alternation and estimation of time during the 2 months of Subterranean Sojourn of a young adult male. $C$. R. Hebd. Seances Acad. Sci. 260, 1259-1262.

Head, G. A., Chatzivlastou, K., Lukoshkova, E. V., Jennings, G. L., and Reid, C. M. (2010). A novel measure of the power of the morning blood pressure surge from ambulatory blood pressure recordings. Am. J. Hypertens. 23, 1074-1081.

Head, G. A., Lukoshkova, E. V., Mayorov, D. N., and Van Den Buuse, M. (2004). Non-symmetrical doublelogistic analysis of 24-h blood pressure recordings in normotensive and hypertensive rats. J. Hypertens. 22, 2075-2085.

Head, G. A., Reid, C. M., and Lukoshkova, E. V. (2005). Nonsymmetrical double logistic analysis of ambulatory blood pressure recordings. J. Appl. Physiol. 98, 1511-1518.

Head, G. A., Reid, C. M., Shiel, L. M., Jennings, G. L., and Lukoshkova, E. V. (2006). Rate of morning increase in blood pressure is elevated in hypertensives. Am. J. Hypertens. 19, 1010-1017.

Holt-Lunstad, J., and Steffen, P. R. (2007). Diurnal cortisol variation is associated with nocturnal blood pressure dipping. Psychosom. Med. 69, 339-343.

Iantorno, M., Pola, R., Schinzari, F., Filice, G., Mettimano, M., Cardillo, C., and Melina, D. (2003). Association between altered circadian blood pressure profile and cardiac endorgan damage in patients with ren- ovascular hypertension. Cardiology 100, 114-119.

Idema, R. N., Gelsema, E. S., Wenting, G. J., Grashuis, J. L., Van Den Meiracker, A. H., Brouwer, R. M., and Man in 'T Veld, A. J. (1992). A new model for diurnal blood pressure profiling. Square wave fit compared with conventional methods. Hypertension 19, 595-605.

Jones, D. W., and Hall, J. E. (2004). Seventh report of the Joint National Committee on Prevention, Detection, Evaluation, and Treatment of High Blood Pressure and evidence from new hypertension trials. Hypertension 43, 1-3.

Kario, K. (2009). Obstructive sleep apnea syndrome and hypertension: mechanism of the linkage and 24$\mathrm{h}$ blood pressure control. Hypertens. Res. 32, 537-541.

Katz, M. E., Simonetta, S. H., Ralph, M. R., and Golombek, D. A. (2008). Immunosuppressant calcineurin inhibitors phase shift circadian rhythms and inhibit circadian responses to light. Pharmacol. Biochem. Behav. 90, 763-768.

Kemper, M. J., Sparta, G., Laube, G. F., Miozzari, M., and Neuhaus, T. J. (2003). Neuropsychologic sideeffects of tacrolimus in pediatric renal transplantation. Clin. Transplant. 17, 130-134.

Kooman, J. P., Christiaans, M. H., Boots, J. M., Van Der Sande, F. M., Leunissen, K. M., and Van Hooff, J. P. (2001). A comparison between office and ambulatory blood pressure measurements in renal transplant patients with chronic transplant nephropathy. Am. J. Kidney Dis. 37, 1170-1176.

Lipkin, G. W., Tucker, B., Giles, M., and Raine, A. E. (1993). Ambulatory blood pressure and left ventricular mass in cyclosporin- and noncyclosporin-treated renal transplant recipients. J. Hypertens. 11, 439-442.

Lusardi, P., Mugellini, A., Preti, P., Zoppi, A., Derosa, G., and Fogari, R. (1996). Effects of a restricted sleep regimen on ambulatory blood pressure monitoring in normotensive subjects. Am. J. Hypertens. 9, 503-505.

Mohri, T., Emoto, N., Nonaka, H., Fukuya, H., Yagita, K., Okamura, H., and Yokoyama, M. (2003). Alterations of circadian expressions of clock genes in Dahl salt-sensitive rats fed a high-salt diet. Hypertension 42, 189-194.

Nelson, W. T. Y., Lee, J. K., and Halberg, F. (1979). Methods for cosinor rhythmometry. Chronobiologia 6, 305-323.

Neter, J., Kutner, M. H., Nachtsheim, C., and Wasserman, W. (1996). "Tests for constancy of error variance," in Applied Linear Statistical Models, 4th Edn (Boston: McGraw-Hill/Irwin), 112-114.

Neuhaus, P., Mcmaster, P., Calne, R. Pichlmayr, R., Otto, G., Williams, R., Bismuth, H., and Groth, C. (1994). Neurological complications in the European multicentre study of FK 506 and cyclosporin in primary liver transplantation. Transpl. Int. 7(Suppl. 1), S27-S31.

Panza, J. A., Epstein, S. E., and Quyyumi, A. A. (1991). Circadian variation in vascular tone and its relation to alpha-sympathetic vasoconstrictor activity. N. Engl. J. Med. 325, 986-990.

Parati, G. (2004). Assessing circadian blood pressure and heart rate changes: advantages and limitations of different methods of mathematical modelling. J. Hypertens. 22, 2061-2064.

Perez-Lloret, S., Aguirre, A. G., Cardinali, D. P., and Toblli, J. E. (2004). Disruption of ultradian and circadian rhythms of blood pressure in nondipper hypertensive patients. Hypertension 44, 311-315.

Portaluppi, F., Waterhouse, J., and Minors, D. (1996). The rhythms of blood pressure in humans. Exogenous and endogenous components and implications for diagnosis and treatment. Ann. N. Y. Acad. Sci. 783 1-9.

Rudic, R. D., and Fulton, D. J. (2009) Pressed for time: the circadian clock and hypertension. J. Appl. Physiol. 107, 1328-1338.

Smolensky, M. H., Hermida, R. C. Castriotta, R. J., and Portaluppi, F. (2007). Role of sleep-wake cycle on blood pressure circadian rhythms and hypertension. Sleep Med. 8, 668-680.

Stanton, A., Cox, J., Atkins, N., O'malley, K., and O'brien, E. (1992). Cumulative sums in quantifying circadian blood pressure patterns. Hypertension 19, 93-101.

Taler, S. J., Textor, S. C., Canzanello, V. J., Schwartz, L., Porayko, M. K., Wiesner, R. H., and Krom, R. A. (2000). Hypertension after liver transplantation: a predictive role for pretreatment hemodynamics and effects of isradipine on the systemic and renal circulations. Am. J. Hypertens. 13, 231-239.
Taler, S. J., Textor, S. C., Canzanello, V. J., Wilson, D. J., Wiesner, R. H., and Krom, R. A. (1995). Loss of nocturnal blood pressure fall after liver transplantation during immunosuppressive therapy. Am. J. Hypertens. 8, 598-605.

Textor, S. C., Canzanello, V. J., Taler, S. J., Wilson, D. J., Schwartz, L. L., Augustine, J. E., Raymer, J. M., Romero, J. C., Wiesner, R. H., and Krom, R. A. (1994). Cyclosporine-induced hypertension after transplantation. Mayo Clin. Proc. 69, 1182-1193.

van de Borne, P., Gelin, M., Van De Stadt, J., and Degaute, J. P. (1993). Circadian rhythms of blood pressure after liver transplantation. Hypertension 21, 398-405.

van den Dorpel, M. A., Van Den Meiracker, A. H., Lameris, T. W., Boomsma, F., Levi, M., Man in 'T Veld, A. J., Weimar, W., and Schalekamp, M. A. (1996). Cyclosporin A impairs the nocturnal blood pressure fall in renal transplant recipients. Hypertension 28, 304-307.

Witte, K., Schnecko, A., Buijs, R. M., Van Der Vliet, J., Scalbert, E., Delagrange, P., Guardiola-Lemaitre, B., and Lemmer, B. (1998). Effects of SCN lesions on circadian blood pressure rhythm in normotensive and transgenic hypertensive rats. Chronobiol. Int. 15, 135-145.

Conflict of Interest Statement: The authors declare that the research was conducted in the absence of any commercial or financial relationships that could be construed as a potential conflict of interest.

Received: 22 November 2011; accepted: 22 February 2012; published online: 16 March 2012.

Citation: Katz ME, Margulis F, Schiavelli $R$, Arias P, Head GA and Golombek DA (2012) Disruption of transitional stages in 24-h blood pressure recording in renal transplant recipients. Front. Neur. 3:35. doi: 10.3389/fneur.2012.00035

This article was submitted to Frontiers in Sleep and Chronobiology, a specialty of Frontiers in Neurology.

Copyright (C) 2012 Katz, Margulis, Schiavelli, Arias, Head and Golombek. This is an open-access article distributed under the terms of the Creative Commons Attribution Non Commercial License, which permits non-commercial use, distribution, and reproduction in other forums, provided the original authors and source are credited. 